\title{
ARTICLE OPEN \\ Bacterial attachment and biofilm formation on surfaces are reduced by small-diameter nanoscale pores: how small is small enough?
}

\author{
Guoping Feng ${ }^{1,3}$, Yifan Cheng ${ }^{1,3}$, Shu-Yi Wang ${ }^{2}$, Diana A Borca-Tasciuc ${ }^{2}$, Randy W Worobo ${ }^{1}$ and Carmen I Moraru ${ }^{1}$
}

BACKGROUND/OBJECTIVES: Prevention of biofilm formation by bacteria is of critical importance to areas that directly affect human health and life including medicine, dentistry, food processing and water treatment. This work showcases an effective and affordable solution for reducing attachment and biofilm formation by several pathogenic bacteria commonly associated with foodborne illnesses and medical infections.

METHODS: Our approach exploits anodisation to create alumina surfaces with cylindrical nanopores with diameters ranging from 15 to $100 \mathrm{~nm}$, perpendicular to the surface. The anodic surfaces were evaluated for attachment by Escherichia coli, Listeria monocytogenes, Staphylococcus aureus and Staphylococcus epidermidis. Cell-surface interaction forces were calculated and related to attachment.

RESULTS: We found that anodic alumina surfaces with pore diameters of 15 and $25 \mathrm{~nm}$ were able to effectively minimise bacterial attachment or biofilm formation by all the microorganisms tested. Using a predictive physicochemical approach on the basis of the extended Derjaguin and Landau, Verwey and Overbeek (XDLVO) theory, we attributed the observed effects largely to the repulsive forces, primarily electrostatic and acid-base forces, which were greatly enhanced by the large surface area originating from the high density, small-diameter pores. We also demonstrate how this predictive approach could be used to optimise different elements of surface topography, particularly pore diameter and density, for further enhancing the observed bacteria-repelling effects.

CONCLUSIONS: We demonstrate that anodic nanoporous surfaces can effectively reduce bacterial attachment. These findings are expected to have immediate, far-reaching implications and commercial applications, primarily in health care and the food industry.

npj Biofilms and Microbiomes (2015) 1, 15022; doi:10.1038/npjbiofilms.2015.22; published online 2 December 2015

\section{INTRODUCTION}

Biofilms are the prevailing lifestyle of bacteria in most natural environments. They consist of microbial communities that usually accumulate at solid-liquid interfaces and are entrapped in a matrix of highly hydrated extracellular polymeric substances. ${ }^{1}$ The quiescent lifestyle of microbial cells living in such a densely packed diffusion barrier is responsible for their high tolerance to environmental stresses. ${ }^{2}$ Cells in biofilms have been deemed 100 to 1,000 times more resistant to antibiotics and disinfecting agents than planktonic cells. ${ }^{3,4}$ Biofilm formation by pathogenic bacteria has deleterious, sometimes fatal consequences, and leads to severe contamination problems in medicine, dentistry, food processing, water treatment and other areas that directly affect human health and life. For instance, it is estimated that approximately $80 \%$ of all medical infections are derived from biofilm growth of pathogens. ${ }^{5}$ Biofilms formed by pathogens in food processing plants are a major culprit in the spread of foodborne diseases, which claim thousands of lives and amount to losses of about $\$ 78$ billion/year in the United States alone. ${ }^{6}$

Most studies attempting to mitigate the effects of biofilms focus on interventions aimed to kill microbial cells in biofilms already present on solid surfaces. ${ }^{7,8}$ However, such strategies have limited efficacy owing to bacterial persistence and resistance in preformed biofilms., ${ }^{9,10}$ Surface modification is emerging as a promising strategy for preventing biofilm formation on abiotic surfaces. There is increasing evidence that bacterial attachment and subsequent biofilm formation are significantly impacted by surface topography. ${ }^{11-13}$ For surfaces with topographic features at the micrometric scale, comparable with the size of prokaryotic cells, cells tend to position themselves such that they maximise contact area with the surface, which favours attachment. ${ }^{11,12}$ Surfaces with topographic features of dimensions much smaller than microbial cells, in the submicrometric or nanometric range, have been reported to inhibit attachment by reducing the contact area between bacteria cells and the surface. ${ }^{11,13}$ In addition, surface topography at the nanoscale can create energetic situations unfavourable for bacterial attachment, and induce repulsive surface-bacteria interaction forces that impair attachment and subsequent biofilm formation. ${ }^{14,15}$ Although most nanostructuring methods available today require cleanroom technologies and are prohibitively expensive for large-scale applications, anodisation is an inexpensive, commercially available electrochemical method that allows relatively easy control of surface features in the nanometre range. ${ }^{12}$

${ }^{1}$ Department of Food Science, Cornell University, Ithaca, NY, USA and ${ }^{2}$ Department of Mechanical, Aerospace and Nuclear Engineering, Rensselaer Polytechnic Institute, Troy, NY, USA.

Correspondence: DA Borca-Tasciuc (borcad@rpi.edu) or CI Moraru (cim24@cornell.edu)

${ }^{3}$ These authors contributed equally to this work.

Received 12 May 2015; revised 31 August 2015; accepted 7 September 2015 
A recent study by our research group showed that anodic alumina surfaces with nanoscale cylindrical pores of diameters smaller than $25 \mathrm{~nm}$ are able to reduce bacterial attachment by the non-pathogenic Escherichia coli and Listeria innocua. ${ }^{15}$ The observed effects were largely attributed to additional repulsive forces contributed by the large surface area of those substrates with a high density of small-diameter pores. The current report builds on these promising findings and tests the ability of anodic alumina surfaces to reduce bacterial attachment for several pathogenic strains. A quantitative prediction of bacteria-surface interaction forces is used to understand how to further optimise surface topographical features and physicochemical properties and thus create surfaces with a stronger ability to prevent bacteria attachment and biofilm formation.

As challenge organisms, four of the most feared pathogens associated with medical, biomedical or food processing environments were selected: E. coli 0157:H7, L. monocytogenes, Staphylococcus aureus and Staphylococcus epidermidis, along with the non-pathogenic E. coli K12. L. monocytogenes can cause illness, death and abortion, and is of particular concern for immuno-compromised individuals and pregnant women. The United States Centers for Disease Control and Prevention estimates that about 2,500 listeriosis and 500 associated deaths occur yearly. ${ }^{16}$ The ubiquitous $L$. monocytogenes can be transmitted through raw foods, the environment, utensils or processing equipment. ${ }^{17-19}$ Infection with $E$. coli 0157:H7 can lead to severe foodborne illness, specifically haemorrhagic diarrhoea and haemolytic uraemic syndrome. ${ }^{16,20,21}$ Contamination with $S$. aureus is the root cause for a range of illnesses, from food poisoning to infections of the skin and soft tissue, to respiratory, bone, joint and endovascular disorders; S. aureus is the most frequently isolated pathogen from wound infections. ${ }^{22,23} \mathrm{~S}$. epidermidis has been associated with bacteremia, catheter-related infection, central nervous system shunt infection, endocarditis, urinary tract infection, surgical site infection and endophthalmitis. ${ }^{24}$

\section{MATERIALS AND METHODS}

\section{Surface fabrication}

Nanoporous aluminium oxide (alumina) surfaces with pore diameters of $15,25,50$ and $100 \mathrm{~nm}$ were prepared by two-step anodisation of high purity aluminium (99.99\%, Alfa Aesar), which has been described in detail before. ${ }^{15,25}$ The aluminium substrate was first subjected to mechanical and electrochemical polishing, with an intermediate annealing process meant to release internal stresses. The polished substrate was immersed in an etchant to remove the thin alumina layer formed during electrochemical polishing. The first anodisation step was carried out at room temperature using a setup similar to that used for electrochemical polishing. The voltage and anodising mixture depended on the pore size. The first porous alumina layer was etched away and a second anodisation step was performed, during which pore growth was initiated from dents left over by the nanopores in the first layer, resulting in regular surface features. ${ }^{25,26}$ Nanosmooth alumina surfaces of $10 \times 10 \times 0.5 \mathrm{~mm}$ (Alfa Aesar, Ward Hill, MA, USA) were used as a control. The nanosmooth control used here had similar surface roughness (root-mean-square roughness $R_{\mathrm{rms}}<1 \mathrm{~nm}$ ), but different surface properties compared with that used in our previous study ${ }^{15}$ (a water contact angle of $39.3 \pm 1.1^{\circ}$ as compared with $67.5 \pm 5.0^{\circ}$ previously).

\section{Bacteria attachment}

Cultures of E. coli 0157:H7 ATCC 43894, E. coli K12, L. monocytogenes 10403 S, S. aureus 9144, S. epidermidis ATCC 35984 were maintained in tryptic soy broth with $20 \%$ (volume/volume) glycerol at $-80^{\circ} \mathrm{C}$. Cultures were reactivated on tryptic soy agar at $37^{\circ} \mathrm{C}$ for $24 \mathrm{~h}$. They were grown in tryptic soy broth for $24 \mathrm{~h}$ and subcultured in tryptic soy broth for $16 \mathrm{~h}$ at $37^{\circ} \mathrm{C}$. The experimental procedure was described in detail before..$^{15}$ Briefly, 16-hour-old cultures of planktonic cells at a diluted concentration of $\sim 10^{7}$ $\mathrm{CFU} / \mathrm{ml}$ were incubated statically for $48 \mathrm{~h}$ with vertically placed anodised alumina surfaces and the nanosmooth control, respectively, at the optimal growth temperature for each bacterial strain, then retrieved and evaluated for bacterial attachment. An incubation time of $48 \mathrm{~h}$ was chosen since it was previously observed that this time point allowed bacteria to attach to the surfaces in sufficient numbers for a meaningful quantitative assessment, but without significant biofilm formation. ${ }^{15}$ Surfaces were placed vertically to reflect true attachment and minimise the effect of cell sedimentation due to gravity. For modelling purposes, the nutritive broth was approximated as a 1:1 type electrolyte solution of ionic strength $0.1 \mathrm{M}$, at $\mathrm{pH} 7$ and a temperature of $310 \mathrm{~K}\left(37^{\circ} \mathrm{C}\right)$.

\section{Confocal laser scanning microscopy}

The surfaces with attached cells were gently removed from the culture and rinsed in sterile saline solution $(0.15 \mathrm{M} \mathrm{NaCl})$, three times, to remove lightly attached cells. The bacterial biomass was labelled with Syto 9 (Molecular Probes Inc., Eugene, OR, USA). A Zeiss 710 confocal laser scanning microscopy equipped with inverted objectives was used to acquire threedimensional images of live bacteria, as described before. ${ }^{15}$ For every type of surface, six replicates (two surfaces per each of three independent experiments) were used. On each sampled surface, at least five randomly selected and evenly spaced fields $\left(338.4 \times 338.4 \mu^{2}\right)$ were scanned. Threedimensional images of biomass matrices were constructed using Volocity (version 5.2.1, PerkinElmer, Waltham, MA, USA).

\section{Biomass quantification}

The total biomass and surface coverage were quantified using COMSTAT, a computer programme designed specifically for this purpose. ${ }^{27}$ A threshold value of 3 was assigned to all the individual image stacks. Quantified parameters were: biomass accumulation $\left(\mu \mathrm{m}^{3} / \mu \mathrm{m}^{2}\right)$, obtained by dividing the overall biomass volume by the substratum area; and layer coverage, given by the percentage of the area occupied by bacteria in each optical layer.

\section{Scanning electron microscopy}

Visualisation of biomass structures was conducted with a Zeiss LEO 1550 field emission scanning electron microscopy, and images acquired with the SmartSEM software (Carl Zeiss Microscopy, LLC, Hamburg, Germany). Surfaces were retrieved at $48 \mathrm{~h}$ and rinsed in saline solution to remove lightly attached cells. The biomass on the surfaces was fixed using $2.5 \%(\mathrm{w} /$ v) glutaraldehyde in $0.05 \mathrm{M}$ sodium cacodylate buffer at $4{ }^{\circ} \mathrm{C}$ for $2 \mathrm{~h}$. Samples were then rinsed in cacodylate buffer three times, $5 \mathrm{~min}$ each time, and subjected to secondary fixation with $1 \%(\mathrm{w} / \mathrm{v})$ osmium tetroxide in cacodylate buffer, for $1 \mathrm{~h}$. The fixated samples were rinsed in cacodylate buffer three times, then dehydrated using gradient ethanol solutions of $25 \%(\mathrm{v} / \mathrm{v}), 50,70,95,100$ and $100 \%$, for 10 min each, followed by critical point drying with carbon dioxide. Dried surfaces were mounted to scanning electron microscopy stubs and coated with evaporated carbon. A voltage of 1 to $5 \mathrm{kV}$ was used, depending on the sample.

\section{Contact angle measurement}

Contact angles of water, glycerol and diiodomethane on both bacterial cell lawns and alumina substrates were determined by the sessile drop method with a Rame-Hart 500 goniometer (Rame-Hart Inc., Succasunna, NJ, USA), as described before. ${ }^{15}$

\section{Surface electric charges}

The zeta potential of the bacterial cells was measured using a Malvern Zetasizer nano-ZS with disposable folded capillary cells (Malvern Instruments, Malvern, UK), as described previously. ${ }^{15}$ Zeta potential of the alumina was taken from Li and Logan.28

\section{Statistical analysis}

One-way analysis of variance and post-analysis-of-variance Tukey's test were used to compare multiple means. All analyses were performed with JMP Pro 11 (SAS Institute, Cary, NJ, USA). The adjusted XDLVO model was constructed and computed using Mathematica 9.0 (Wolfram, Champaign IL, USA). 


\section{RESULTS}

Biomass accumulation and structure on nanotopographic anodic alumina surfaces

Consistent with our previous observations, ${ }^{15}$ presence of bacteria on the anodic surfaces with pores of 15 and $25 \mathrm{~nm}$ was largely reduced compared with the anodised surfaces with larger pore sizes, for all strains. Both visual observations (Figure 1) and the quantitative biomass evaluation (Figure 2) showed that the extent of biomass accumulation varied considerably among the tested microorganisms. For E. coli K12, E. coli O157:H7 and L. monocytogenes, a thin layer of uniformly distributed cells was observed on all surfaces, while for S. aureus and S. epidermidis, both prolific biofilm formers, biomass accumulation was much more significant, particularly on the surfaces with pore diameters above $50 \mathrm{~nm}$ (Figure 2, left panels). At $48 \mathrm{~h}$, the biomass accumulation for $E$. coli $0157: \mathrm{H} 7$ on the nanosmooth, 15, 25, 50 and $100 \mathrm{~nm}$ surfaces was $0.32 \mu^{3} / \mu^{2}, 0.36 \mu \mathrm{m}^{3} / \mu \mathrm{m}^{2}$, $0.47 \mu \mathrm{m}^{3} / \mu \mathrm{m}^{2}, \quad 0.86 \mu \mathrm{m}^{3} / \mu \mathrm{m}^{2}$ and $1.51 \mu \mathrm{m}^{3} / \mu \mathrm{m}^{2}$, respectively. The biomass accumulation for $E$. coli $\mathrm{K} 12$ was slightly lower than for E. coli 0157:H7. For L. monocytogenes, the biomass accumulation was about one order of magnitude higher than for the $E$. coli strains, ranging from $4.27 \mu^{3} / \mu \mathrm{m}^{2}$ on $15 \mathrm{~nm}$ surfaces to $16.40 \mathrm{~mm}^{3} / \mu \mathrm{m}^{2}$ on $100 \mathrm{~nm}$ surfaces. Biomass accumulation by S. aureus and S. epidermidis followed a similar trend, but was much more pronounced compared with the two E. coli strains and L. monocytogenes. S. aureus had the lowest biomass accumulation on the $15 \mathrm{~nm}$ surface $\left(16.91 \mathrm{\mu m}^{3} / \mu \mathrm{m}^{2}\right)$ and the highest on the $100 \mathrm{~nm}$ surface $\left(28.19 \mu \mathrm{m}^{3} / \mu^{2}\right)$. S. epidermidis had the lowest biomass on the $25 \mathrm{~nm}$ surface $\left(25.54 \mu \mathrm{m}^{3} / \mu \mathrm{m}^{2}\right)$ and the highest on the nanosmooth control $\left(50.56 \mu \mathrm{m}^{3} / \mu \mathrm{m}^{2}\right)$. The thickness of the S. epidermidis biomass often exceeded $100 \mu \mathrm{m}$, several times higher than for any of the other strains tested. For the $100 \mathrm{~nm}$ surfaces, 48-h biomass accumulation by $S$. epidermidis had the clear traits of a dense biofilm and often included microcolonies (Figure 1, bottom right). As a note, the nanosmooth controls used in the current work were different than those used in our previous study. ${ }^{15}$ This resulted in slightly different levels of biomass accumulation than previously reported for the nanosmooth alumina controls, but the attachment trends were maintained.

Overall, the trend in biomass accumulation on the anodic alumina surfaces by all strains agrees with our previous observations. ${ }^{15}$ Owing to some variability in the data, not all differences in biomass accumulation among surface types were statistically significant $(P<0.05)$. Nonetheless, a closer analysis of the biomass structure further substantiates the differences among surface types. We generated vertical three-dimensional confocal laser scanning microscopy scans of the biomass structure for all anodic alumina surfaces, in $1 \mu \mathrm{m}$ vertical increments, and calculated the surface coverage by biofilm for each layer. The right panels in Figure 2 show surface coverage at different distances from the substratum for the four pathogenic strains. For all strains, biofilm surface coverage was lowest for the 15 and $25 \mathrm{~nm}$ surfaces, and highest for the 50 and $100 \mathrm{~nm}$ surfaces. For the weak biofilm former E. coli O157:H7, maximum coverage ranged between $1 \%$ on the $15 \mathrm{~nm}$ surfaces and $4 \%$ on the $100 \mathrm{~nm}$ surfaces. The most striking differences in coverage among surfaces were observed for $L$. monocytogenes, for which maximum coverage on the surfaces with small pore sizes (about 20\%) was

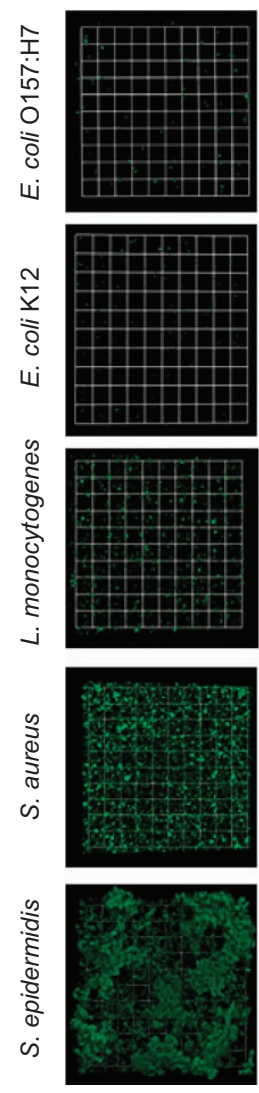

Nanosmooth
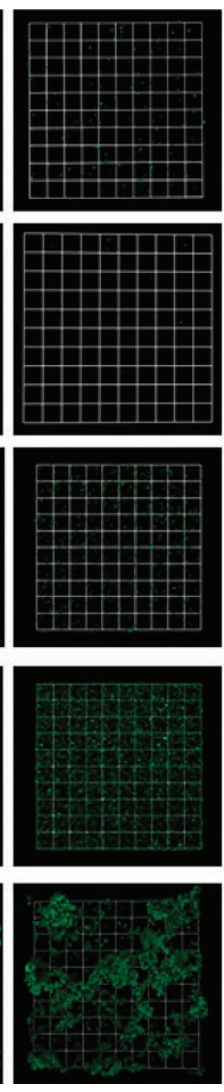

15
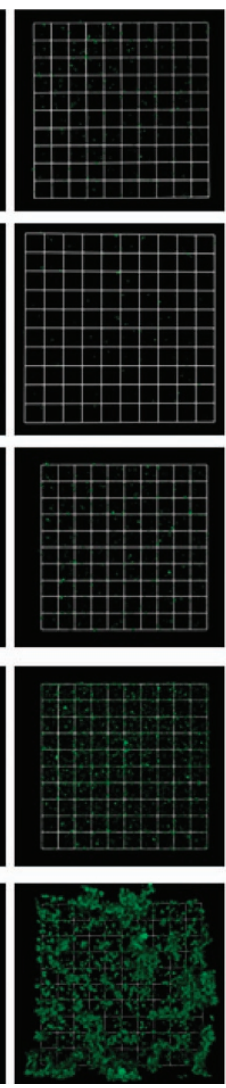

25
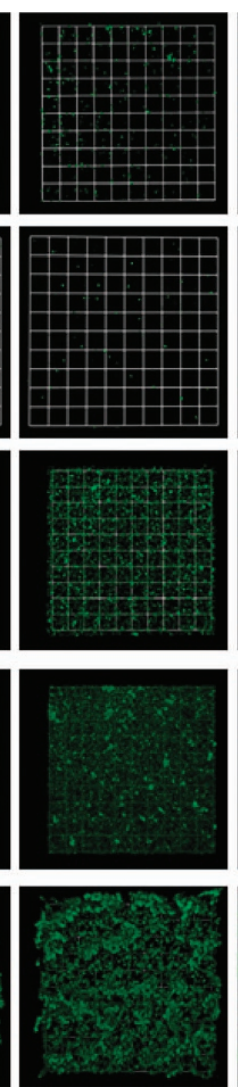

$50 \mathrm{~nm}$
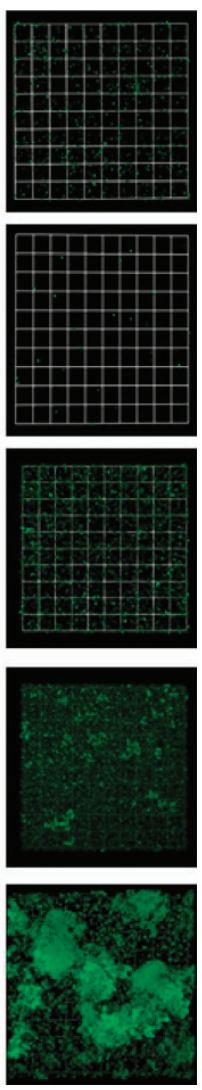

$100 \mathrm{~nm}$

Figure 1. Constructed confocal laser scanning microscopy (CLSM) three-dimensional images of 48-hour-old biofilms of $E$. coli O157:H7, E. coli $\mathrm{K} 12$, L. monocytogenes, S. aureus and S. epidermidis on nanosmooth alumina (control) and anodised surfaces of 15, 25, 50 and 100 nm pore diameter. The presented images have biomass accumulation close to the average for their surface type, so that images are representative. Scale units (small grid) are $34 \mu \mathrm{m}$ in length. 

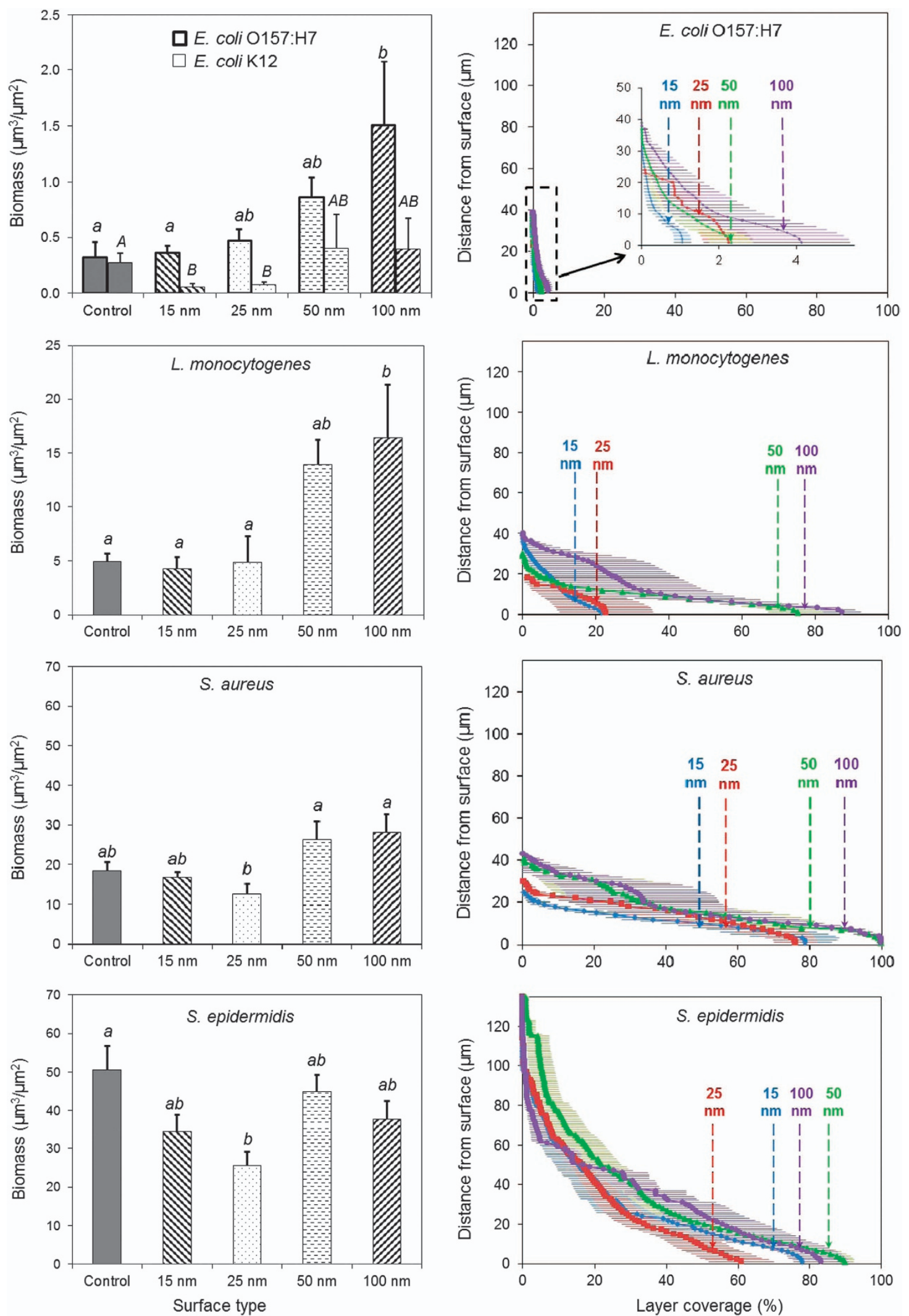

Figure 2. Differences in biomass accumulation over $48 \mathrm{~h}$ by E. coli, L. monocytogenes, S. aureus and S. epidermidis among alumina surfaces. Left panels: quantified average values of biomass $\left(\mu \mathrm{m}^{3} / \mu \mathrm{m}^{2}\right.$ ) accumulated on nanosmooth alumina (control), and anodised surfaces of $15,25,50$ and $100 \mathrm{~nm}$ pore diameter. Values not connected by the same letter are statistically different from each other $(P<0.05)$. Error bars represent standard error of mean. Right panels: layer coverage as a function of distance from the surface. Error bars represent standard errors. 
S. aureus
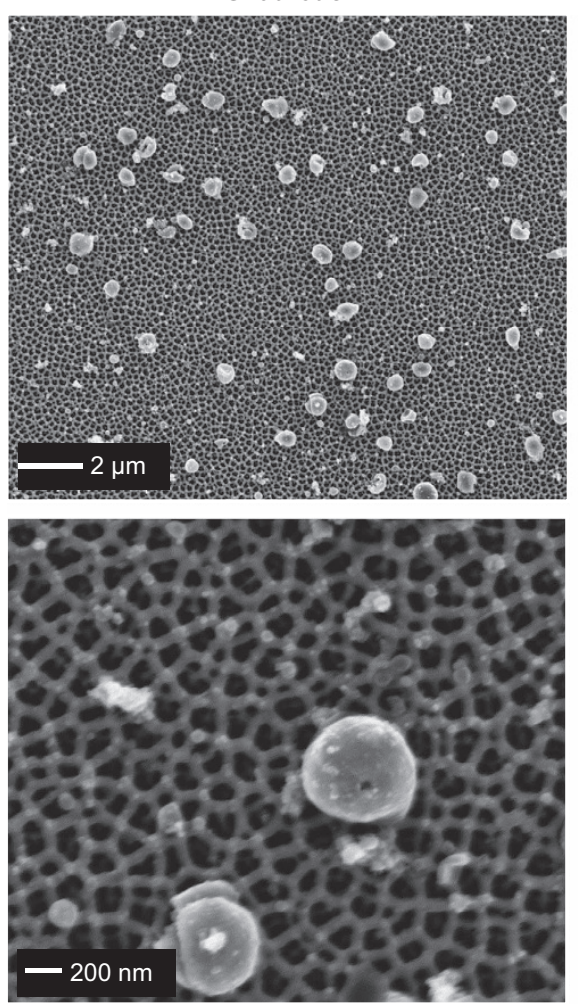

S. epidermidis
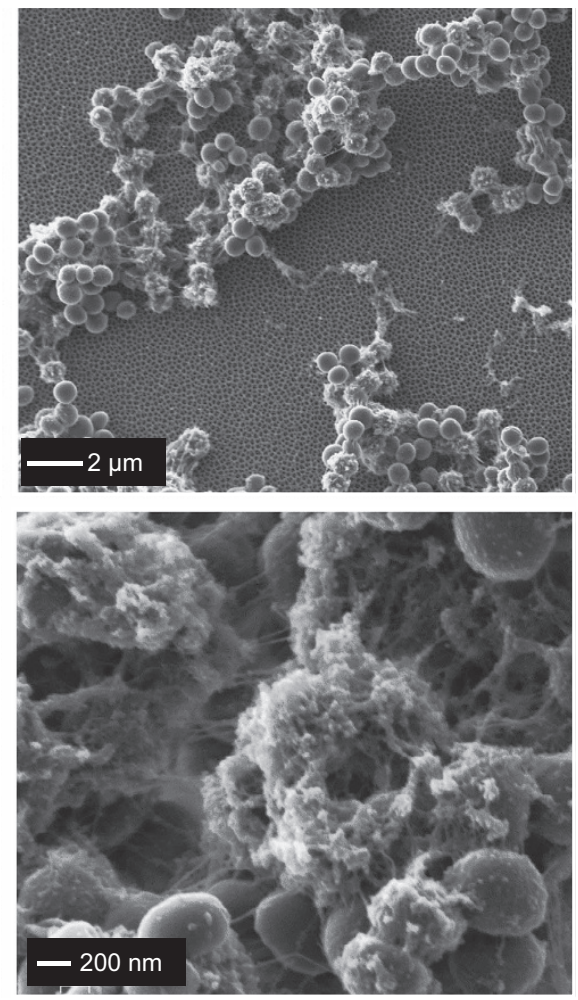

Figure 3. Scanning electron microscopy images of S. aureus (left images) and S. epidermidis cells (right images) at low magnification (upper) and high magnification (lower) after $48 \mathrm{~h}$ contact time with anodic alumina surfaces with $100 \mathrm{~nm}$ pore diameter. Bottom right image shows S. epidermidis cells entrapped in a matrix of extracellular material.
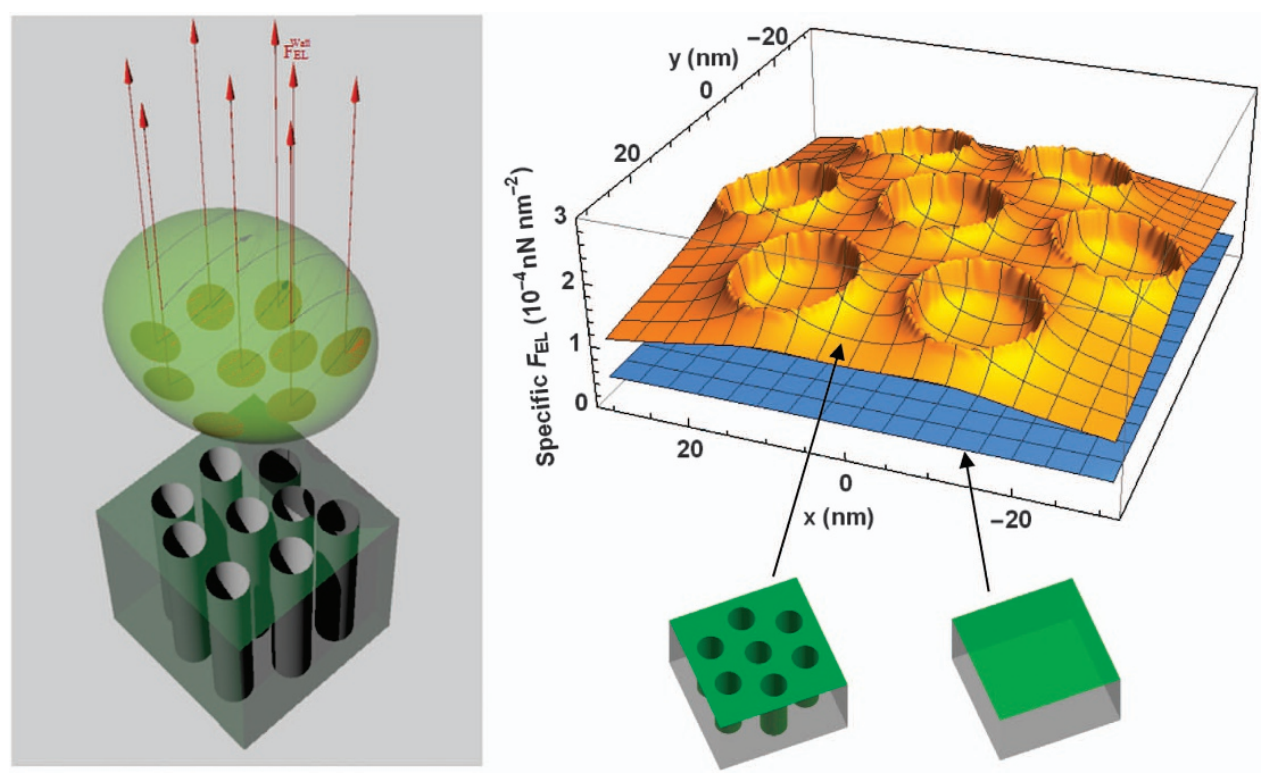

Figure 4. Electrostatic repelling force field exerted on bacterial cells by the nanoporous surface. Left: schematic representation of the electrostatic repelling forces exerted on a bacterial cell by the nanopores located directly underneath the cell. Right: spatial distribution of the electrostatic repulsive force field exerted on E. coli O157:H7 cells by an anodic alumina surface with cylindrical pores of $15 \mathrm{~nm}$ diameter and 2,259 $\mathrm{nm}$ pore depth (top plot) compared with a smooth alumina surface (bottom plot), at a cell-surface separation distance of $0.2 \mathrm{~nm}$. The surface components contributing to the force fields are illustrated in green.

four-fold smaller than on surfaces with larger pore sizes (about 80\%). The prolific biofilm former S. epidermidis had high coverage on all surfaces, ranging from a low $61 \%$ on the $25 \mathrm{~nm}$ surfaces to a high $90 \%$ on the $50 \mathrm{~nm}$ surfaces. For the other strains, significant coverage was found mostly within a few micrometres from the surface, whereas for S. epidermidis, biomass coverage was very high, even tens of micrometres from the surface.

To further investigate the biomass structure, we conducted scanning electron microscopy visualisation of the surfaces with the largest pore size $(100 \mathrm{~nm})$, which had either the largest or 


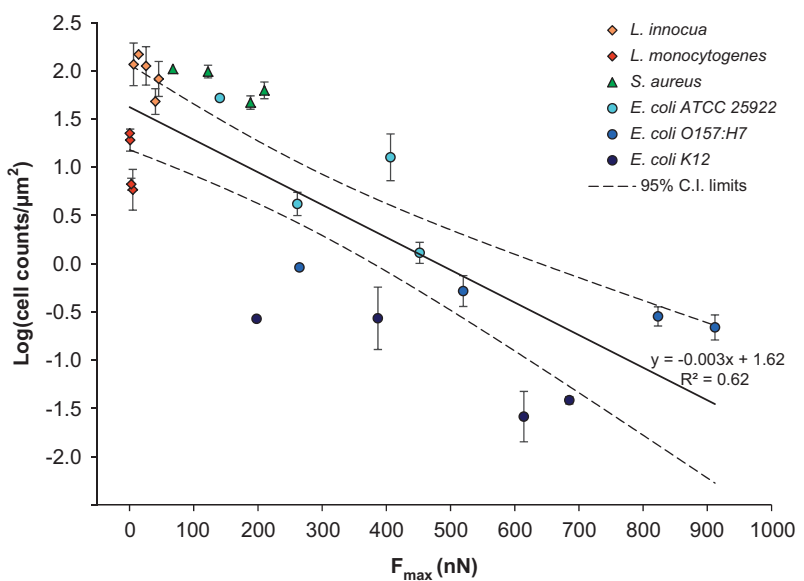

Figure 5. Bacteria cell counts in the biofilm per unit area of surface as a function of $F_{\text {max }}$ after $48 \mathrm{~h}$ of incubation. The regression equation and $R^{2}$ value are for all the strains pooled together. Error bars represent standard errors. C.I., confidence interval.

second largest biomass accumulation. It should be noted that the scanning electron microscopy images cannot be used for quantitative purposes, since some cell detachment from the surfaces may have occurred during scanning electron microscopy sample preparation. Figure 3 shows scanning electron microscopy micrographs for S. aureus (left) and S. epidermidis (right). For S. aureus, a single layer of cells was generally observed, with minimal amount of extracellular material. S. epidermidis on the other hand showed substantial, vertically grown biofilms, with a high density of bacterial cells tightly intertwined in a matrix of extracellular material, which is very typical of $S$. epidermidis biofilms. ${ }^{30}$ The tremendous biofilm forming ability of S. epidermidis has been attributed to a large extent to the contribution of specific biological factors, mainly the polysaccharide intercellular adhesin encoded by the intercellular adhesion (ica) locus. ${ }^{31,32}$ All other bacterial strains showed a single layer of cells, and no significant indication of extracellular substances (images not shown). On the basis of this corroborated evidence, in the subsequent discussion, we will refer to the biomass accumulation as attached cells for all the strains except $S$. epidermidis, for which a biofilm was formed.

Overall, this study clearly shows that the small nanoscale pore anodic alumina surfaces can effectively limit cell attachment and biofilm formation by a range of bacteria relevant for medical, biomedical and food processing applications. The trend was similar regardless of Gram-positive or Gram-negative status, rod or coccus shape, or the ability of the cells to express appendages under the conditions tested.

Bacterial attachment correlates with surface-bacteria interaction forces

Previously, we found strong evidence of a correlation between attachment by two bacteria strains and the overall cell-surface interaction force, calculated using the extended Derjaguin and Landau, Verwey and Overbeek (XDLVO) theory. ${ }^{15}$ One important prediction made using this model was the maximum repulsive force that a bacterial cell needs to overcome and come into direct contact with a surface, termed ' $F_{\max }$ '. We were able to show that as $F_{\max }$ increased, the number of bacteria attached to a surface decreased. ${ }^{15}$ Therefore, the XDLVO predictive approach was used to estimate bacteria-surface interaction forces for the strains tested in this study. Predictions were limited to those strains that did not show biofilm formation over the $48 \mathrm{~h}$ duration of the test: E. coli $0157: \mathrm{H} 7$, E. coli K12, L. monocytogenes and S. aureus. S. epidermidis was not included in these predictions, because the presence of a significant amount of extracellular material did not allow an accurate estimation of the number of cells (Figure 4).

Cell properties required by the model were determined experimentally as described in the Materials and Methods section, and their measured values are summarised in Supplementary Table S1. When applying the XDLVO model, the following simplifying assumptions were made: (i) all cells were assumed to be spherical in shape, and for rod-shaped bacteria (E. coli and Listeria) an equivalent radius was calculated; (ii) surfaces have a fully wetting (Wenzel) behaviour ${ }^{33}$ and (iii) each surface was assumed to be an infinite planar surface relative to a bacterial cell. The surface properties are included in Supplementary Table S2, and the other constants used in the calculations are in Supplementary Table S3.

The overall interaction force between a bacterium cell and the anodic alumina surfaces, $F_{\text {Total }}^{\text {XDVO }}$, was calculated by the vector addition of three force components: electrostatic force $\left(F_{\mathrm{EL}}\right)$, acidbase interaction force $\left(F_{\mathrm{AB}}\right)$, and Lifshitz-van der Waals interaction force $\left(F_{\mathrm{LW}}\right)$. As all the forces are affected by the nanopores underneath the cells, they were adjusted to take into consideration this effect (denoted as 'Adj'):

$$
F_{\text {Total }}^{\mathrm{XDLVO}}=F_{\mathrm{LW}}^{\mathrm{Adj}}+F_{\mathrm{AB}}^{\mathrm{Adj}}+F_{\mathrm{EL}}^{\mathrm{Adj}}
$$

The full expression of equation (1) and the derivation of its components are presented in detail in our previous study. ${ }^{15}$ Briefly, the Lifshitz-van der Waals interaction force between the cells and the surfaces $\left(F_{\mathrm{LW}}^{\mathrm{Adj}}\right)$ was calculated using the retarded Hamaker expression. The acid-base interaction force $\left(F_{A B}^{A d j}\right)$, which incorporated the effect of surface energy, was calculated using the extended Young equation. ${ }^{34}$ As acid-base interactions are short range interactions, it was considered that only the top rim of the vertical surface of the cylindrical nanopores $(2 \mathrm{~nm}$ from the surface) effectively contributes to the acid-base interaction. This portion of the internal surface of the cylindrical pores was approximated as a ring of hemispheres distributed uniformly along the circumference. The repulsion force exerted on one bacterium by the total number of cylindrical walls underneath that cell was calculated considering the pore diameter and surface porosity, as well as the radius of the effective circular interaction area for each type of bacteria.

The electrostatic interaction force $\left(F_{\mathrm{EL}}^{\mathrm{Adj}}\right)$ was calculated for each pore and its surrounding area and the total force was determined by multiplying the value of this force by the number of pores underneath one bacterium cell. An example of the contribution of nanoscale topographical features to the magnitude and spatial distribution of interaction forces, the specific $F_{\mathrm{EL}}$ (electrostatic force per unit area, or 'electrostatic pressure') exerted on a bacterial cell by the surface, plotted as a function of the radial distance from the centre of a cylindrical pore, is shown in Supplementary Figure S1. Regardless of pore size, the vertical walls of the cylindrical pores from the porous anodic surfaces contributed to an increase in the electrostatic force compared with that generated by a smooth surface of similar chemistry. Figure 4 shows both a schematic representation of the total electrostatic forces acting on a E. coli O157:H7 cell (left), and the field of electrostatic forces contributed by a surface area equivalent to a hexagonal array of $15 \mathrm{~nm}$ pores (right), at a cell-surface separation distance where electrostatic forces are significant for this bacterial strain. The force field plot on the right clearly shows that the repulsion exerted by the $15 \mathrm{~nm}$ surface greatly exceed the repulsion by the nanosmooth control surface for $E$. coli O157:H7, which is consistent with the biomass accumulation shown in Figure 1 and Figure 2. Similar plots can be generated for the other forces.

The total cell-surface interaction force was then calculated as a function of the cell-surface separation distance (Supplementary 


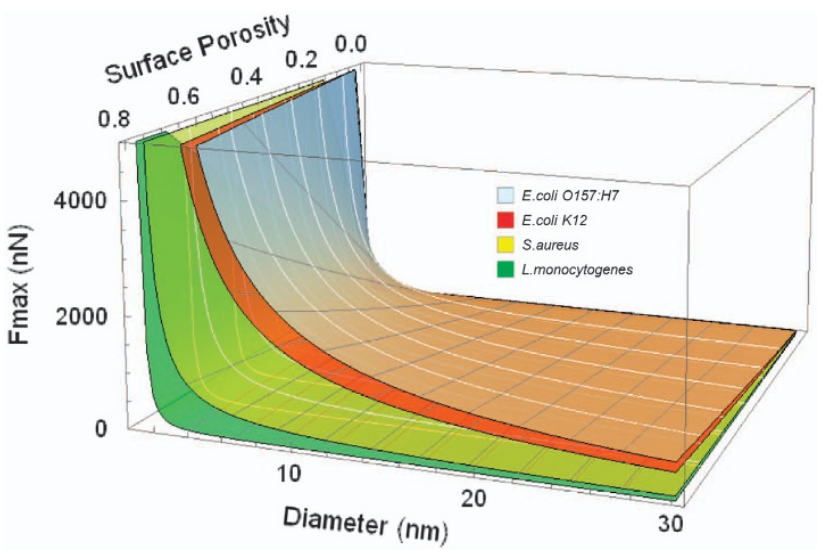

Figure 6. Predicted values of the maximum repelling cell-surface interaction force as a function of pore diameter and surface porosity of the alumina anodic surfaces for E. coli O157:H7, E. coli K12, S. aureus and L. monocytogenes.

Figure S2). In close proximity of the surface, ranging from fractions of a nanometre to several nanometres, depending on the bacterial strain, the bacterium-surface interaction force was attractive for all surface-strain pairs, owing to the short range attractive Lifshitz-van der Waals forces. At separation distances beyond a few nanometres, the repulsive electrostatic and acid-base forces become dominant for all the strains. For the anodic surfaces with the smallest pores, this contribution is particularly significant owing to the large number of cylindrical pores per surface area. Consequently, the total repelling force $\left(F_{\text {Total }}^{\mathrm{XDLV}}\right)$ is particularly high for the $15 \mathrm{~nm}$ and $25 \mathrm{~nm}$ pore surfaces, which have a high density of vertical pores per unit surface area.

$F_{\text {max }}$ for all bacteria-anodic surface pairs used in the study was plotted against the cell counts (Figure 5), calculated on the basis of the biomass volume and the measured cell size (Supplementary Table S1). The data points for two non-pathogenic strains (L. innocua and E. coli ATCC 25922) from our previous work ${ }^{15}$ were also included in this analysis. This plot allowed several important observations. First, for all the strains, the greater the magnitude of the energy barrier, represented by the repulsive $F_{\text {max }}$ the fewer cells accumulated on the surface. A linear correlation between $F_{\max }$ and the number of attached cells was obtained for each strain. As only four data points per strain were available, and because of the variability of some data points, not all correlations were statistically significant. For the strains tested in this study, the values for the coefficient of determination were as follows: $R^{2}=0.743$ for $S$. aureus, $R^{2}=0.864$ for $L$. monocytogenes, $R^{2}=0.815$ for $E$. coli $\mathrm{K} 12$ and $R^{2}=0.998$ for $E$. coli O157:H7. Figure 5 also illustrates a clustering of bacteria from different species. The two Listeria strains (diamond symbols) showed the strongest dependence on $F_{\max }$ which means that small increases in the repulsive force can be extremely effective in reducing the attachment by these bacteria to the anodic surfaces. The three E. coli strains (circle symbols), while showing a good correlation between attachment and $F_{\text {max }}$ were less sensitive to the magnitude of $F_{\max }$ compared with Listeria. S. aureus (triangle symbols) had a behaviour intermediate between Listeria and $E$. coli. The similar response to $F_{\max }$ of bacteria from the same species is not only indicative of the role of biological factors in attachment, but also shows that the physicochemical approach used here is able to reflect these differences.

The other very important observation is that a statistically significant $(P<0.001)$ linear correlation between $F_{\max }$ and the number of attached cells from all bacterial strains was obtained. The solid line in Figure 5 represents the linear regression line, and the dotted lines represent the 95\% confidence interval range for the linear regression. The confidence interval accounts for both the variance of the experimental data and the uncertainty in the numerical coefficients of the linear regression. The $95 \%$ confidence interval lines are the closest in the middle of the $F_{\max }$ range, and further apart towards the ends of the $F_{\max }$ range, which indicates that the uncertainty of the prediction increases for both very low and very high attachment.

\section{DISCUSSION}

Bacterial attachment to abiotic surfaces and subsequent biofilm formation are complex processes, controlled by the interplay between biological factors, such as secretion of extracellular materials by the bacteria, ${ }^{29}$ bacterial appendages and other cell surface structures that can contribute to bacterial sensing of the surface, ${ }^{9}$ and physicochemical factors, such as surface topography, surface charge and surface energy. ${ }^{14}$ As it is practically impossible to alter the properties of naturally occurring bacteria and make them less likely to attach to abiotic surfaces, the more feasible approach to tackle biofouling is to reduce the propensity of the abiotic surfaces to bacterial attachment, by altering their surface chemistry and topography.

The correlation between bacteria attachment and surface topography has been investigated for a long time. Commonly used surface roughness parameters such as average and rootmean-square roughness describe only the height variation of the surface, but not the spatial distribution or the shape of surface features. ${ }^{35}$ Donoso et al. ${ }^{36}$ demonstrated that neither parameter is a reliable predictor for the interfacial area from which surfacebased forces (i.e., $F_{\mathrm{AB}}$ and $F_{\mathrm{EL}}$ ) arise, as the relationship between these parameters and interfacial area is not monotonic. These results explain at least, in part, why despite the volume of work in this area, the mechanisms by which surface roughness and topography modulate attachment remain largely unclear, especially at a scale smaller than the dimension of a bacterial cell. 28,35

The present study shows that attachment by E. coli $0157: \mathrm{H} 7$, E. coli K12, L. monocytogenes, S. aureus and S. epidermidis was impaired on anodic alumina surfaces with pore sizes of 15 and $25 \mathrm{~nm}$ in diameter as compared with surfaces with larger pores, and in some cases with the nanosmooth control surfaces (Figure 1). The visual confocal laser scanning microscopy observations were validated by quantitative assessment of the biofilm matrices (Figure 2).

This study also allowed us to make several very useful observations regarding the effect of abiotic surface properties on bacterial attachment. To date, many biofouling studies used surface energy values calculated from apparent contact angles as a predictor of attachment. Several studies suggest that highly hydrophobic surfaces, which have water contact angles higher than $90^{\circ}$, have a lower propensity for microbial attachment and biofouling. ${ }^{37}$ Nonetheless, the conventional hydrophobichydrophilic dichotomic categorisation of surfaces and bacterial cells has been proved insufficient to make accurate predictions about bacterial attachment. Hook et al. ${ }^{38}$ found no correlation between bacterial attachment and water contact angles for 496 polymeric materials. Rather, they suggested that surface chemical groups dictated the propensity of bacterial attachment onto polymer surfaces rather than water contact angle alone. Our results also show that neither hydrophobicity nor chemistry alone can be used as a predictor for attachment. Despite the fact that all porous surfaces used in our study had similar chemistry, the measured contact angles of three probe liquids varied notably among the surfaces (Supplementary Figure S3). Furthermore, the surfaces that were most effective against bacterial attachment had the smallest contact angles in all the three liquids and a clear hydrophilic behaviour, whereas surfaces with the largest pores were more hydrophobic, yet allowed higher attachment by 
bacteria. To understand the change in contact angle with pore size, it is important to consider the fundamental factors that dictate the apparent contact angles, namely the intrinsic (Young's) contact angle of a liquid droplet on an ideal (rigid, flat, chemically homogeneous, insoluble, nonreactive) solid surface, and surface topography, which can enhance both hydrophobic and hydrophilic reactions. ${ }^{39,40}$ The trends in Supplementary Figure S3 agree with the Wenzel relation, which dictates that surface topography on hydrophilic surfaces (water contact angle $<90^{\circ}$ ) enhances their apparent hydrophilicity. ${ }^{40}$ For alumina surfaces under consideration here, this was likely owing to the capillary effects caused by the cylindrical pores. ${ }^{41}$

A quantitative prediction of the propensity of bacteria to attach onto surfaces is not straightforward. In this work, such a prediction was obtained by using the comprehensive XDLVO model, which considers the role of cell-surface physicochemical interaction forces in attachment. This model has been used before, with varied degrees of success, in describing the interaction between colloidal particles and patterned surfaces, ${ }^{42,43}$ or the interaction between microbial cells and surfaces. ${ }^{44-46}$ One element lacking in previous applications of this model is that contributions from surface elements perpendicular to the surface were not considered. Taking this into account allowed us to explain the extraordinary bacteria-repelling effect of the 15 and $25 \mathrm{~nm}$ alumina surfaces. Specifically, this effect is attributed to the vertical sidewalls of the densely distributed cylindrical pores, which exerted additional electrostatic repulsion and acid-base repulsive forces on the bacterial cells found in the proximity of the surfaces.

The fact that the correlation between cell-surface interaction force, represented by $F_{\max }$ and attachment holds across several bacteria strains (Figure 5 ) is of considerable importance in that, despite the different cell wall structure, shape, presence or absence of cellular appendages, the adjusted XDLVO model is capable of predicting the trends in bacteria attachment with reasonable accuracy. This is also very relevant from a practical perspective. Although this study has been conducted on individual strains, in both natural and man-made environments, bacteria are present in multi-microbial communities. The overall correlation between $F_{\max }$ and attachment found here indicates that an increase in the repulsive force will be able to reduce attachment by multiple bacteria, albeit to different degrees.

As an example of how this model can be used as a design tool, we generated predictions of changes in cell-surface interaction forces induced by changes in the elements of surface topography. Our calculations show that pore diameter has a tremendous effect on the repulsive forces, with $F_{\max }$ increasing exponentially as pore diameter decreases. Increasing surface porosity at a fixed pore diameter results in a proportional increase in $F_{\max }$, while pore depth does not seem to have a significant effect beyond several tens of nanometres. Figure 6 illustrates how changing pore diameter and surface porosity affects $F_{\max }$ for all bacteria strains used in this study, except $S$. epidermidis. What is remarkable is that the effect of surface topography is similar for all microorganisms, even if the magnitude of $F_{\max }$ varies among the different strains. This is extremely meaningful from a practical perspective, because it indicates that further decreasing the pore size and increasing the surface porosity will improve the anti-attachment ability of anodic surfaces.

The findings of this study are of high importance, as they demonstrate a science based, yet relatively simple and practical way to prevent attachment and subsequent biofilm formation by diverse pathogenic, as well as non-pathogenic bacteria.

\section{ACKNOWLEDGEMENTS}

This work made use of the Cornell Center for Materials Research (CCMR) Shared Facilities, supported through the NSF MRSEC program (DMR-1120296), Cornell
University Biotechnology Resource Center (BRC) Imaging Facility, with support from NIH 1S10RR025502-01, and the Cornell Nanobiotechnology Center (NBTC), supported by NSF (Agreement No. ECS-9876771). The authors thank Penny Burke (NBTC), Teresa Porri (NBTC), Malcolm Thomas (CCMR), John Grazul (CCMR) and Carol Bayles (BRC) for their competent assistance. The help from Shujuan Huang of Rensselaer Polytechnic Institute with the fabrication of anodic surfaces is also acknowledged. This project was supported by USDA-NIFA (Project 65210-20024-11).

\section{CONTRIBUTIONS}

GF and YC are equal contributors to the work. GF has executed the attachment study and evaluation of bacterial attachment, and initiated the manuscript. YC has conducted the surface property characterisation for the anodic surfaces and bacterial cells, the XDLVO modelling and had a significant contribution to writing the manuscript. S-YW optimised the anodisation conditions and fabricated the anodic alumina surfaces. RWW provided microbiological expertise to the team, contributed to experimental design and data analyses and interpretation, and manuscript editing DAB-T and CIM are the senior authors of the paper, have conceived the work and hypotheses, defined the objectives and experimental approaches, and have equally contributed to formulating the conclusions and writing the manuscript.

\section{COMPETING INTERESTS}

The authors declare no conflict of interest.

\section{REFERENCES}

1 Flemming H-C, Wingender J. The biofilm matrix. Nat Rev Microbiol 2010; 8 : 623-633.

2 Costerton JW, Stewart PS, Greenberg EP. Bacterial biofilms: a common cause of persistent infections. Science 1999; 284: 1318-1322.

3 Smith K, Hunter IS. Efficacy of common hospital biocides with biofilms of multidrug resistant clinical isolates. J Med Microbiol 2008; 57: 966-973.

4 Stewart PS, William Costerton J. Antibiotic resistance of bacteria in biofilms. Lancet 2001; 358: 135-138.

5 Harro JM, Peters BM, O'May GA, Archer N, Kerns P, Prabhakara R et al. Vaccine development in Staphylococcus aureus: taking the biofilm phenotype into consideration. FEMS Immunol Med Microbiol 2010; 59: 306-323.

6 Scharff RL. Economic burden from health losses due to foodborne illness in the United States. J Food Prot 2012; 75: 123-131.

7 Kolodkin-Gal I, Romero D, Cao S, Clardy J, Kolter R, Losick R. D-amino acids trigger biofilm disassembly. Science 2010; 328: 627-629.

8 Feng G, Klein MI, Gregoire S, Singh AP, Vorsa N, Koo H. The specific degree-ofpolymerization of A-type proanthocyanidin oligomers impacts Streptococcus mutans glucan-mediated adhesion and transcriptome responses within biofilms. Biofouling 2013; 29: 629-640.

9 Hori K, Matsumoto S. Bacterial adhesion: from mechanism to control. Biochem Eng J 2010; 48: 424-434.

10 Bridier A, Briandet R, Thomas V, Dubois-Brissonnet F. Resistance of bacterial biofilms to disinfectants : a review. Biofouling 2011; 26: 1017-1032.

11 Hsu L, Fang J, Borca-Tasciuc D, Worobo R, Moraru Cl. The effect of micro- and nanoscale topography on the adhesion of bacterial cells to solid surfaces. Appl Environ Microbiol 2013; 79: 2703-2712.

12 Whitehead KA, Colligon J, Verran J. Retention of microbial cells in substratum surface features of micrometer and sub-micrometer dimensions. Colloids Surf $B$ Biointerfaces 2005; 41: 129-138.

13 Friedlander RS, Vlamakis H, Kim P, Khan M, Kolter R, Aizenberg J. Bacterial flagella explore microscale hummocks and hollows to increase adhesion. Proc Natl Acad Sci USA 2013; 110: 5624-5629.

14 Anselme K, Davidson P, Popa AM, Giazzon M, Liley M, Ploux L. The interaction of cells and bacteria with surfaces structured the nanometre scale. Acta Biomater 2010; 6: 3824-3846.

15 Feng G, Cheng Y, Wang S-Y, Hsu LC, Feliz Y, Borca-Tasciuc DA et al. Alumina surfaces with nanoscale topography reduce attachment and biofilm formation by Escherichia coli and Listeria spp. Biofouling 2014; 30: 1253-1268.

16 Mead PS, Slutsker L, Dietz V, McCaig LF, Bresee JS, Shapiro C et al. Food-related illness and death in the United States. Emerg Infect Dis 1999; 5: 607-625.

17 Giovannacci I, Ragimbeau C, Queguiner S, Salvat G, Vendeuvre JL, Carlier V et al. Listeria monocytogenes in pork slaughtering and cutting plants use of RAPD, PFGE and PCR-REA for tracing and molecular epidemiology. Int J Food Microbiol 1999; 53: $127-140$.

18 Miettinen MK, Björkroth KJ, Korkeala HJ. Characterization of Listeria monocytogenes from an ice cream plant by serotyping and pulsed-field gel electrophoresis. Int J Food Microbiol 1999; 46: 187-192. 
19 Vogel BF, Huss HH, Ojeniyi B, Ahrens P, Gram L. Elucidation of Listeria monocytogenes contamination routes in cold-smoked salmon processing plants detected by DNA-based typing methods. Appl Environ Microbiol 2001; 67: 2586-2595.

20 Beuchat LR. Ecological factors influencing survival and growth of human pathogens on raw fruits and vegetables. Microbes Infect 2002; 4: 413-423.

21 Karch H, Tarr Pl, Bielaszewska M. Enterohaemorrhagic Escherichia coli in human medicine. Int J Med Microbiol 2005; 295: 405-418.

22 Lowy FD. Staphylococcus aureus infections. N Engl J Med 1998; 339: 520-532.

23 Giacometti A, Cirioni O, Schimizzi AM, Del Prete MS, Barchiesi F, D'Errico MM et al. Epidemiology and microbiology of surgical wound infections. J Clin Microbiol 2000; 38: 918-922.

24 Huebner J, Goldmann DA. Coagulase-negative staphylococci: role as pathogens. Ann Rev Med 1999; 50: 223-236.

25 Jessensky O, Müller F, Gösele U. Self-organized formation of hexagonal pore arrays in anodic alumina. Appl Phys Lett 1998; 72: 1173-1175.

26 Masuda H, Yada K, Osaka A. Self-Ordering of cell configuration of anodic porous alumina with large-size pores in phosphoric acid solution. Jpn J Appl Phys 1998; 37: L1340-L1342.

27 Heydorn A, Nielsen AT, Hentzer M, Sternberg C, Givskov M, Ersbøll BK et al. Quantification of biofilm structures by the novel computer program COMSTAT. Microbiology 2000; 146: 2395-2407.

28 Li B, Logan BE. Bacterial adhesion to glass and metal-oxide surfaces. Colloid Surf $B$ 2004; 36: 81-90.

29 Reisner A, Haagensen JAJ, Schembri MA, Zechner EL, Molin S. Development and maturation of Escherichia coli K-12 biofilms. Mol Microbiol 2003; 48: 933-946.

30 Evans E, Brown MRW, Gilbert P. Iron chelator, exopolysaccharide and protease production in Staphylococcus epidermidis: a comparative study of the effects of specific growth rate in biofilm and planktonic culture. Microbiology 1994; 140: 153-157.

31 Heilmann C, Schweitzer O, Gerke C, Vanittanakom N, Mack D, Götz F. Molecula basis of intercellular adhesion in the biofilm-forming Staphylococcus epidermidis. Mol Microbiol 1996; 20: 1083-1091.

32 Mack D, Fischer W, Krokotsch A, Leopold K, Hartmann R, Egge $\mathrm{H}$ et al. The intercellular adhesin involved in biofilm accumulation of Staphylococcus epidermidis is a linear $\beta-1,6$-linked glucosaminoglycan: purification and structural analysis. J Bacteriol 1996; 178: 175-183.

33 Ran C, Ding G, Liu W, Deng Y, Hou W. Wetting on nanoporous alumina surface: transition between Wenzel and Cassie states controlled by surface structure. Langmuir 2008; 24: 9952-9955.

34 Van Oss CJ. Acid-base interfacial interactions in aqueous media. Colloids Surf $A$ Physicochem Eng Asp 1993; 78: 1-49.
35 Crawford RJ, Webb HK, Truong VK, Hasan J, Ivanova EP. Surface topographical factors influencing bacterial attachment. Adv Colloid Interfac 2012; 179-182: $142-149$.

36 Donoso MG, Méndez-Vilas A, Bruque JM, González-Martin ML. On the relationship between common amplitude surface roughness parameters and surface area: Implications for the study of cell-material interactions. Int Biodeter Biodegr 2007; 59: $245-251$.

37 Mérian T, Goddard JM. Advances in nonfouling materials: perspectives for the food industry. J Agric Food Chem 2012; 60: 2943-2957.

38 Hook AL, Chang C, Yang J, Luckett J, Cockayne A, Atkinson S et al. Combinatorial discovery of polymers resistant to bacterial attachment. Nat Biotechnol 2012; 30: 868-875.

39 Gao L, McCarthy TJ. How Wenzel and cassie were wrong. Langmuir 2007; 23: 3762-3765.

40 Wenzel R. Resistance of solid surfaces. J Ind Eng Chem 1936; 28: 988-994.

41 Ran C, Ding G, Liu W, Deng Y, Hou W. Wetting on nanoporous alumina surface: transition between Wenzel and Cassie states controlled by surface structure. Langmuir 2008; 24: 9952-9955.

42 Hoek EMV, Bhattacharjee S, Elimelech M. Effect of membrane surface roughness on colloid-membrane DLVO interactions. Langmuir 2003; 19 : 4836-4847.

43 Martines $\mathrm{E}$, Csaderova L, Morgan H, Curtis ASG, Riehle MO. DLVO interaction energy between a sphere and a nano-patterned plate. Colloid Surf A 2007; 318: 45-52.

44 Chen Y, Harapanahalli AK, Busscher HJ, Norde W, van der Mei HC. Nanoscale cell wall deformation impacts long-range bacterial adhesion forces on surfaces. Appl Environ Microbiol 2014; 80: 637-643.

45 Chrysikopoulos CV, Syngouna VI. Attachment of bacteriophages MS2 and ФX174 onto kaolinite and montmorillonite: extended-DLVO interactions. Colloids Surf $B$ Biointerfaces 2012; 92: 74-83.

46 Chia TWR, Nguyen VT, McMeekin T, Fegan N, Dykes GA. Stochasticity of bacterial attachment and its predictability by the extended Derjaguin-Landau-VerweyOverbeek theory. Appl Environ Microbiol 2011; 77: 3757-3764.

This work is licensed under a Creative Commons Attribution 4.0 International License. The images or other third party material in this article are included in the article's Creative Commons license, unless indicated otherwise in the credit line; if the material is not included under the Creative Commons license, users will need to obtain permission from the license holder to reproduce the material. To view a copy of this license, visit http://creativecommons.org/licenses/ by/4.0/
}

Supplementary Information accompanies the paper on the npj Biofilms and Microbiomes website (http://www.nature.com/npjbiofilms) 